\title{
A Unified Description of Particles, Strings and Branes in Clifford Spaces and p-brane/polyparticle Duality
}

\author{
Carlos Castro
}

December, 2014

Center for Theoretical Studies of Physical Systems, Clark Atlanta University, Atlanta, GA. 30314; perelmanc@hotmail.com

\begin{abstract}
It is proposed how the Extended Relativity Theory in $C$-spaces (Clifford spaces) allows a unified formulation of point particles, strings, membranes and $p$-branes, moving in ordinary target spacetime backgrounds, within the description of a single polyparticle moving in $C$-spaces. The degrees of freedom of the latter are provided by Clifford polyvector-valued coordinates (antisymmetric tensorial coordinates). A correspondence between the $p$-brane ( $p$-loop) wave functional "Schroedinger-like" equations of Ansoldi-Aurilia-Spallucci and the polyparticle wave equation in $C$ spaces is found via the polyparticle/p-brane duality/correspondence. The crux of exploiting this correspondence is that it might provide another unexplored avenue to quantize $p$-branes (a notoriously difficult and unsolved problem) from the more straightforward quantization of the polyparticle in $C$-spaces, even in the presence of external interactions. We conclude with some comments about the compositeness nature of the polyvector-valued coordinate operators in terms of ordinary $p$-brane coordinates via the evaluation of $n$-ary commutators.
\end{abstract}

Keywords : Clifford algebras; Extended Relativity in Clifford Spaces; Strings; Branes; $M$-theory; polyparticle/ $p$-brane duality; loop wave functional equations; Born-Dirac oscillator; $n$-ary commutators.

After more than four decades of string theory research (see [1] for more recent books on string theory) there are still many questions pending. Among some of the problems are: The quantization program of $p$-branes (for $p \geq 2$ ) remains unsolved; the foundation of string theory ( $M$-theory, $F$-theory) from a fundamental physical principle (like general covariance and the equivalence principle in General Relativity) has not been envisioned yet; the fundamental variables of $M$ - theory, $F$-theory and the underlying origin of string 
dualities are unknown; the formulation of a unified description of all $p$-branes on a single footing has not been constructed. The aim of this preliminary work is to shed some light onto some of these problems by recurring to the Extended Relativity Theory in $C$-spaces (Clifford spaces). Recent avenues of research in string theory are currently being pursued based on generalized geometries like Exceptional and Double Field Theories (see [2], [3] for most recent references). We must not confuse the C-spaces associated with closed 3forms in Double Field Theories [3] with the $C$-spaces (Clifford-spaces) in this work. They are very different generalized geometries.

In the past years, the Extended Relativity Theory in $C$-spaces (Clifford spaces) and Clifford-Phase spaces were developed [4], [5]. The Extended Relativity theory in Cliffordspaces (C-spaces) is a natural extension of the ordinary Relativity theory whose generalized coordinates are Clifford polyvector-valued quantities which incorporate the lines, areas, volumes, and hyper-volumes degrees of freedom associated with the collective dynamics of particles, strings, membranes, $p$-branes (closed p-branes) moving in a D-dimensional target spacetime background. $C$-space Relativity should permit us to study the dynamics of all (closed) $p$-branes, for different values of $p$, on a unified footing, as we intend to show.

Our theory has 2 fundamental parameters : the speed of a light $c$ and a length scale which can be set equal to the Planck length. The role of "photons" in $C$-space is played by tensionless branes. An extensive review of the Extended Relativity Theory in Clifford spaces can be found in [4]. The polyvector valued coordinates $X^{\mu}, X^{\mu_{1} \mu_{2}}, X^{\mu_{1} \mu_{2} \mu_{3}}, \ldots$ are now linked to the basis vectors generators $\gamma^{\mu}$, bi-vectors generators $\gamma_{\mu} \wedge \gamma_{\nu}$, tri-vectors generators $\gamma_{\mu_{1}} \wedge \gamma_{\mu_{2}} \wedge \gamma_{\mu_{3}}, \ldots$ of the Clifford algebra, including the Clifford algebra unit element (associated to a scalar coordinate).

These polyvector valued coordinates can be interpreted as the quenched-degrees of freedom of an ensemble of $p$-loops associated with the dynamics of closed $p$-branes, for $p=0,1,2, \ldots, D-1$, embedded in a target $D$-dimensional spacetime background. $C$-space is parametrized not only by 1 -vector coordinates $X^{\mu}$ but also by the 2 -vector coordinates $X^{\mu_{1} \mu_{2}}, 3$-vector coordinates $X^{\mu_{1} \mu_{2} \mu_{3}}, \ldots$, called also holographic coordinates by [13], since they describe the holographic projections of 1-loops, 2-loops, 3-loops,..., onto the coordinate planes. By $p$-loop we mean a closed $p$-brane of topology $S^{p}$. In particular, a 1-loop is closed string. When $\mathbf{X}$ is the Clifford-valued coordinate corresponding to the $C l(1,3)$ algebra in four-dimensions it can be decomposed as

$$
\mathbf{X}=X 1+X^{\mu} \gamma_{\mu}+X^{\mu \nu} \gamma_{\mu} \wedge \gamma_{\nu}+X^{\mu \nu \rho} \gamma_{\mu} \wedge \gamma_{\nu} \wedge \gamma_{\rho}+X^{\mu \nu \rho \tau} \gamma_{\mu} \wedge \gamma_{\nu} \wedge \gamma_{\rho} \wedge \gamma_{\tau}
$$

where we have omitted combinatorial numerical factors for convenience in the expansion of eq-(1). To avoid introducing powers of a length parameter $L$ (like the Planck scale $L_{p}$ ), in order to match physical units in the expansion of the polyvector $\mathbf{X}$ in eq-(1), we can set it to unity to simplify matters. The component $X$ is the Clifford-scalar component of the polyvector-valued coordinate $\mathbf{X}$ and $d \Sigma$ is the infinitesimal $C$-space proper "time" interval

$$
(d \Sigma)^{2}=(d X)^{2}+d X_{\mu} d X^{\mu}+d X_{\mu \nu} d X^{\mu \nu}+\ldots
$$


that is invariant under $C l(1,3)$ transformations and which are the Clifford-algebraic extensions of the $S O(1,3)$ Lorentz transformations [4]. One should emphasize that $d \Sigma$ is not equal to the proper time Lorentz-invariant interval $d \tau$ in ordinary spacetime $(d \tau)^{2}=$ $g_{\mu \nu} d X^{\mu} d X^{\nu}=d X_{\mu} d X^{\mu}$. Generalized Lorentz transformations (poly-rotations) in flat $C$ spaces were discussed in [4], [8]. A detailed discussion on Clifford geometric algebras and polyvectors can be found in [6], [7] . An extensive analysis of the $C$-space generalized Lorentz transformations and their physical implications can be found in [9], [10].

An ordinary $p$-brane moving in a $D$-dim flat target background spacetime spans a $p+1$-dimensional world volume and the Nambu-Goto action written in terms of the Nambu-Poisson bracket is given by

$$
\begin{gathered}
S=T \int d^{p+1} \sigma \sqrt{\left|\operatorname{det} \partial_{a} X^{\mu} \partial_{b} X^{\nu} \eta_{\mu \nu}\right|}= \\
T \int d^{p+1} \sigma \sqrt{\left(\left\{X^{\mu_{1}}, X^{\mu_{2}}, X^{\mu_{3}}, \ldots, X^{\mu_{p+1}}\right\}\right)^{2}}
\end{gathered}
$$

$T$ is the $p$-brane tension whose units are $(\text { mass })^{p+1} . X^{\mu}\left(\sigma^{a}\right)$ are the embedding functions of the $p+1$-dim world volume of the $p$-brane into the $D$-dim target spacetime background $(D \geq p+1)$. The world volume coordinates are $\sigma=\left(\sigma^{1}, \sigma^{2}, \cdots, \sigma^{p+1}\right)$.

Let us generalize the action (3) to the $C$-space case when the world volume and target space coordinates are both Clifford-valued. Given the $2^{d}$ polyvector valued world indices

$$
\sigma^{A}=s, \quad \sigma^{a}, \quad \sigma^{a_{1} a_{2}}, \quad \cdots, \quad \sigma^{a_{1} a_{2} \ldots a_{d}}
$$

and the $2^{D}$ polyvector valued target space indices

$$
X^{M}=X, X^{\mu}, X^{\mu_{1} \mu_{2}}, \cdots, X^{\mu_{1} \mu_{2} \ldots \mu_{D}}
$$

with $D \geq d$ one can write the analog of the expression for a Nambu-Poisson bracket in $C$-space as [4]

$$
\epsilon^{A_{1} A_{2} \ldots A_{2^{d}}} \frac{\partial X^{M_{1}}}{\partial \sigma^{A_{1}}} \frac{\partial X^{M_{2}}}{\partial \sigma^{A_{2}}} \cdots \frac{\partial X^{M_{2^{d}}}}{\partial \sigma^{A_{2^{d}}}}
$$

In general each polyvector valued coordinate component $X^{M}\left(\sigma^{A}\right)$ is a function of all the $2^{d}$ polyvector valued world indices $\sigma^{A}$. The expression (6) simplifies considerably in the very special case when

$$
\begin{gathered}
X=X(s), X^{\mu}=X^{\mu}\left(\sigma^{a}\right), X^{\mu_{1} \mu_{2}}=X^{\mu_{1} \mu_{2}}\left(\sigma^{a_{1} a_{2}}\right), \ldots, X^{\mu_{1} \mu_{2} \cdots \mu_{d}}=X^{\mu_{1} \mu_{2} \cdots \mu_{d}}\left(\sigma^{a_{1} a_{2} \cdots a_{d}}\right), \\
X^{\mu_{1} \mu_{2} \cdots \mu_{d+1}}=X^{\mu_{1} \mu_{2} \cdots \mu_{d+2}}=\ldots=X^{\mu_{1} \mu_{2} \cdots \mu_{D}}=0
\end{gathered}
$$

The above conditions of eq-(7) basically describe grade-preserving maps from the Cliffordvalued world manifold to the target Clifford-valued $C$-space. It comprises of maps such that points are mapped to points; areas to areas; volumes to volumes ... and where one freezes to zero the polyvector valued target coordinates whose grade exceeds the dimension $d$ (of the world manifold an whose associated $C$-space is $2^{d}$-dim). 
In this case the determinant-like expression (6) factorizes as follows

$$
\begin{aligned}
& \frac{\partial X}{\partial s}\left(\epsilon^{a_{1} a_{2}} \ldots a_{d} \frac{\partial X^{\mu_{1}}}{\partial \sigma^{a_{1}}} \frac{\partial X^{\mu_{2}}}{\partial \sigma^{a_{2}}} \cdots \frac{\partial X^{\mu_{d}}}{\partial \sigma^{a_{d}}}\right) \quad\left(\epsilon^{\left[a_{1} b_{1}\right]\left[a_{2} b_{2}\right]} \ldots \frac{\partial X^{\mu_{1} \nu_{1}}}{\partial \sigma^{a_{1} b_{1}}} \frac{\partial X^{\mu_{2} \nu_{2}}}{\partial \sigma^{a_{2} b_{2}}} \cdots\right) \\
& \left(\epsilon^{\left[a_{1} b_{1} c_{1}\right]\left[a_{2} b_{2} c_{2}\right] \ldots} \frac{\partial X^{\mu_{1} \nu_{1} \rho_{1}}}{\partial \sigma^{a_{1} b_{1} c_{1}}} \frac{\partial X^{\mu_{2} \nu_{2} \rho_{2}}}{\partial \sigma^{a_{2} b_{2} c_{2}}} \ldots\right)\left(\epsilon^{\left[a_{1} b_{1} c_{1} d_{1}\right]\left[a_{2} b_{2} c_{2} d_{2}\right]} \ldots \frac{\partial X^{\mu_{1} \nu_{1} \rho_{1} \tau_{1}}}{\partial \sigma^{a_{1} b_{1} c_{1} d_{1}}} \frac{\partial X^{\mu_{2} \nu_{2} \rho_{2} \tau_{2}}}{\partial \sigma^{a_{2} b_{2} c_{2} d_{2}}} \cdots\right) \ldots
\end{aligned}
$$

It is convenient to introduce an ordering of indices to avoid having to introduce factorial numerical factors. For the bivector coordinates $\sigma^{a_{1} b_{1}}, \sigma^{a_{2} b_{2}}, \cdots$ one requires to have $a_{1}<b_{1} ; a_{2}<b_{2} ; \cdots$. For the trivector coordinates $\sigma^{a_{1} b_{1} c_{1}}, \sigma^{a_{2} b_{2} c_{2}}, \cdots$ one requires to have $a_{1}<b_{1}<c_{1} ; a_{2}<b_{2}<c_{2} ; \cdots$, etc. Similar ordering prescription applies for the $X^{\mu_{1} \nu_{1}}, X^{\mu_{1} \nu_{1} \rho_{1}}, \cdots$ target $C$-space polyvector valued coordinates. The generalized version of the epsilon symbols ensures that no polyvector-valued indices are repeated.

A remark is in order. One could also view the induced metric $H_{A B}=$ $\partial_{A} X^{M} \partial_{B} X^{N} G_{M N}$ on the $2^{d}$-dim Clifford-valued world volume as a hyper-matrix but the construction (if possible) of the hyper-determinant of the $C$-space metric (a hyper matrix) is a more difficult problem [11], [12]. For this reason we shall not pursue this road at the moment to build generalized $p$-brane actions in $C$-spaces based on Nambu-Goto actions associated with the square roots of hyper-determinants.

Let us proceed. For example, when $d=2$ and $D \geq d$, eq-(8) is given by

$$
\frac{\partial X}{\partial s}\left(\epsilon^{a_{1} a_{2}} \frac{\partial X^{\mu_{1}}}{\partial \sigma^{a_{1}}} \frac{\partial X^{\mu_{2}}}{\partial \sigma^{a_{2}}}\right) \frac{\partial X^{\mu_{1} \nu_{1}}}{\partial \sigma^{12}}
$$

The term $\frac{\partial X}{\partial s}$ corresponds effectively to the motion of a point particle parametrized by the variable $s$ and moving in one-dimension described by the coordinate $X$. The term $\epsilon^{a_{1} a_{2}} \frac{\partial X^{\mu_{1}}}{\partial \sigma^{a_{1}}} \frac{\partial X^{\mu_{2}}}{\partial \sigma^{a_{2}}}$ is the standard Poisson bracket $\left\{X^{\mu_{1}}, X^{\mu_{2}}\right\}$ with respect to the variables $\sigma^{1}, \sigma^{2}$ and associated with the motion of a string in a $D$-dimensional background. The term $\frac{\partial X^{\mu_{1} \nu_{1}}}{\partial \sigma^{12}}$ corresponds effectively to the motion of a point particle parametrized by the (bivector) variable $\sigma^{12}$ and moving in a $\frac{D(D-1)}{2}$-dimensional background described by the (bivector) coordinates $X^{\mu_{1} \nu_{1}}$.

The analog of the Nambu-Goto action would now be

$$
\begin{array}{r}
S=\kappa \int d s d \sigma^{1} d \sigma^{2} d \sigma^{12} \sqrt{\left(\frac{\partial X}{\partial s}\right)^{2}} \sqrt{\left(\left\{X^{\mu_{1}}, X^{\mu_{2}}\right\}\right)^{2}} \sqrt{\left(\frac{\partial X^{\mu_{1} \nu_{1}}}{\partial \sigma^{12}}\right)^{2}}= \\
\kappa \int d s \sqrt{\left(\frac{\partial X}{\partial s}\right)^{2}} \int d \sigma^{1} d \sigma^{2} \sqrt{\left(\left\{X^{\mu_{1}}, X^{\mu_{2}}\right\}\right)^{2}} \int d \sigma^{12} \sqrt{\left(\frac{\partial X^{\mu_{1} \nu_{1}}}{\partial \sigma^{12}}\right)^{2}}= \\
\kappa \int d X \int d \sigma^{1} d \sigma^{2} \sqrt{\left(\left\{X^{\mu_{1}}, X^{\mu_{2}}\right\}\right)^{2}} \int \sqrt{\left(d X^{\mu_{1} \nu_{1}}\right)^{2}}
\end{array}
$$

Concluding, the action (10) factorizes and collectively describes a point particle moving in one-dim; a string moving in $D$-dim, and a point particle moving in $\frac{D(D-1)}{2}$-dim. Furthermore, one should notice that one has a product of terms instead of a summation of individual actions. 
The conditions of eq- $(7)$ in the more general case $(D \geq d>2)$ describe richer dynamics . Taking into account that a $p$-brane spans a $p+1$ dimensional world volume one has that the second term in eq-(8) describes the standard Nambu-Poisson bracket associated to a $d$-1-brane (spanning a $d$-dim world volume) moving in a $D$-dim target background. The third term describes effectively a $\frac{d(d-1)}{2}-1$-brane moving in a $\frac{D(D-1)}{2}$-dim target background. The fourth term describes effectively a $\frac{d(d-1)(d-2)}{3 !}-1$-brane moving in a $\frac{D(D-1)(D-2)}{3 !}$-dim target background; and so forth. The final term $\left(\partial X^{\mu_{1} \mu_{2} \cdots \mu_{d}} / \partial \sigma^{a_{1} a_{2} \cdots a_{d}}\right)$ corresponds effectively to the motion of a point particle parametrized by the (highest grade polyvector) variable $\sigma^{123 \cdots d}$ and moving in a $\frac{D(D-1)(D-2) \cdots(D-d)}{d !}$-dimensional background described by the (polyvector) coordinates $X^{\mu_{1} \mu_{2} \cdots \mu_{d}}$.

When the conditions of eq-(7) are imposed on the target $C$-space polyvector valued coordinates the number of degrees of freedom $N$ (when $D \geq d$ ) is given by

$$
N=\left(\begin{array}{l}
D \\
0
\end{array}\right)+\left(\begin{array}{l}
D \\
1
\end{array}\right)+\left(\begin{array}{l}
D \\
2
\end{array}\right)+\ldots+\left(\begin{array}{l}
D \\
d
\end{array}\right) \geq\left(\begin{array}{l}
d \\
0
\end{array}\right)+\left(\begin{array}{l}
d \\
1
\end{array}\right)+\left(\begin{array}{l}
d \\
2
\end{array}\right)+\ldots+\left(\begin{array}{l}
d \\
d
\end{array}\right)=2^{d}
$$

hence the number of transverse degrees of freedom is $N-2^{d} \geq 0$. When $D=d$ one has $N=2^{D}=2^{d}$ and the number of transverse degrees of freedom is zero as expected. Therefore, by choosing $D>d$ one will have non-trivial dynamics since the number of transverse degrees of freedom are not zero.

To sum up, the action associated with the expression in eq-(8) is defined to be

$$
S=\kappa \int d s d \sigma^{1} d \sigma^{2} \ldots d \sigma^{12} d \sigma^{13} \ldots d \sigma^{123} d \sigma^{124} \ldots d \sigma^{123 \ldots d} \sqrt{\Delta}
$$

where $\Delta$ is the square of the expression given by eq-(8) and effectively describes a collective ensemble of points and $p$-branes, for certain specific values of $p$, and each moving in different target space dimensions as discussed above.

We will describe now how a polyparticle in $C$-space may have a correspondence with a nested hierarchy of point particles, strings, membranes and $p$-branes in ordinary space. Let us begin by recalling the infinitesimal interval displacement in $C$ space

$$
\begin{aligned}
(d \Sigma)^{2}=d X_{M} d X^{M}= & (d X)^{2}+L^{-2} d X_{\mu} d X^{\mu}+L^{-4} d X_{\mu_{1} \mu_{2}} d X^{\mu_{1} \mu_{2}}+\ldots+ \\
& L^{-2 D} d X_{\mu_{1} \mu_{2} \ldots . . \mu_{D}} d X^{\mu_{1} \mu_{2} \ldots . \mu_{D}}
\end{aligned}
$$

$X$ is the Clifford-scalar part of the Clifford-valued coordinate $X^{M}$. The values of $M$ range from $1,2, \cdots, 2^{D}$. $\Sigma$ and $X$ are both taken to be dimensionless. The polyparticle dynamics is parametrized by the $C$-space proper time variable $\Sigma$ such that the polyvector valued coordinates describing the motion of the polyparticle in $C$-space are determined by the $2^{D}$ functions $X^{M}=X^{M}(\Sigma)$.

When $n=p+1$, the $p$-brane/polyparticle duality/correspondence is defined as follows

$$
L^{2 n}\left\{X^{\mu_{1}}, X^{\mu_{2}}, \ldots, X^{\mu_{n}}\right\}_{\sigma^{a}}^{2} \leftrightarrow\left(\frac{d X^{\mu_{1} \mu_{2} \ldots \ldots \mu_{n}}}{d \Sigma}\right)^{2}
$$




$$
\frac{1}{L^{n}} \int d \Sigma \sqrt{\left(\frac{d X^{\mu_{1} \mu_{2} \ldots . \mu_{n}}}{d \Sigma}\right)^{2}} \leftrightarrow T_{n} \int d^{n} \sigma \sqrt{\left\{X^{\mu_{1}}, X^{\mu_{2}}, \ldots, X^{\mu_{n}}\right\}^{2}}
$$

$T_{n}$ is the $n-1$-brane tension and whose physical units are $(\text { mass })^{n}=(\text { length })^{-n}$. The values of $n$ range from $1,2, \cdots, D$. For $n=1$ one has the point particle action parametrized by the time-like variable $\sigma^{1}$ of the world line in ordinary spacetime.

$$
S=m \int d \sigma^{1} \sqrt{\left(\frac{d X^{\mu}}{d \sigma^{1}}\right)^{2}}
$$

For $n=2$ one has the string action

$$
S=T_{2} \int d \sigma^{1} d \sigma^{2} \sqrt{\left(\left\{X^{\mu_{1}}, X^{\mu_{2}}\right\}\right)^{2}}, \quad\left\{X^{\mu_{1}}, X^{\mu_{2}}\right\}=\epsilon^{a_{1} a_{2}} \frac{\partial X^{\mu_{1}}}{\partial \sigma^{a_{1}}} \frac{\partial X^{\mu_{2}}}{\partial \sigma^{a_{2}}}, a=1,2
$$

where $\sigma^{1}, \sigma^{2}$ are the temporal and spatial coordinates of the worldsheet, respectively; and so forth. For a $p$-brane whose world volume is $n=p+1$-dim one writes the $p$-brane action given by the right hand side of eq-(14b) in terms of the Nambu-Poisson bracket

$$
\left\{X^{\mu_{1}}, X^{\mu_{2}}, \ldots, X^{\mu_{n}}\right\}=\epsilon^{a_{1} a_{2}} \ldots a_{p+1} \frac{\partial X^{\mu_{1}}}{\partial \sigma^{a_{1}}} \frac{\partial X^{\mu_{2}}}{\partial \sigma^{a_{2}}} \ldots \frac{\partial X^{\mu_{p+1}}}{\partial \sigma^{a_{p+1}}}, a=1,2,3, \cdots, p+1
$$

One should note that we are using the epsilon symbol in defining all the above brackets. One could have used the epsilon symbol only in the highest grade case, corresponding to the case when $p+1=D$, and defined the lower grade brackets in terms of an auxiliary number of antisymmetric tensor fields $\omega^{a_{1} a_{2} \ldots .}$ of different ranks if one performed the derivatives with respect to all the $\sigma^{a}$ variables $\sigma^{1}, \sigma^{2}, \cdots, \sigma^{D}$. For instance

$$
\begin{gathered}
\left\{X^{\mu_{1}}, X^{\mu_{2}}\right\}=\omega^{a_{1} a_{2}} \frac{\partial X^{\mu_{1}}}{\partial \sigma^{a_{1}}} \frac{\partial X^{\mu_{2}}}{\partial \sigma^{a_{2}}}, \quad a=1,2,3, \cdots, D \\
\left\{X^{\mu_{1}}, X^{\mu_{2}}, X^{\mu_{3}}\right\}=\omega^{a_{1} a_{2} a_{3}} \frac{\partial X^{\mu_{1}}}{\partial \sigma^{a_{1}}} \frac{\partial X^{\mu_{2}}}{\partial \sigma^{a_{2}}} \frac{\partial X^{\mu_{3}}}{\partial \sigma^{a_{3}}}, \quad a=1,2,3, \cdots, D
\end{gathered}
$$

etc, ..... However this multisymplectic approach will complicate matters since one must satisfy the (generalized) Jacobi identities (fundamental identities) which will constrain the functional form of the auxiliary number of antisymmetric tensor fields $\omega^{a_{1} a_{2} \ldots . . .}$. For this reason, to simplify matters we define the brackets solely in terms of epsilon symbols as shown above in eqs- $(16,17)$. In this way we have a nested hierarchy of point particles, strings, membranes and $p$-branes in ordinary spacetime. The sequence of variables is nested as follows $\sigma^{1} \subset\left(\sigma^{1}, \sigma^{2}\right) \subset\left(\sigma^{1}, \sigma^{2}, \sigma^{3}\right) \subset \cdots \subset\left(\sigma^{1}, \sigma^{2}, \cdots, \sigma^{D}\right)$.

A realization of the duality conditions in eq-(14a) can be simply realized when both sides of eq-(14a) are equal to a constant. Since the right hand side of eq-(14a) depends on $\Sigma$ and the left hand side depends on $\sigma^{a}$ an equality is possible when both sides are equal to a constant. In particular, the simplest choice to attain this equality is when 


$$
X^{1}=c_{1} \sigma^{1}, \quad X^{2}=c_{2} \sigma^{2}, X^{3}=c_{3} \sigma^{3}, X^{n}=c_{n} \sigma^{n}, \ldots, X^{D}=c_{D} \sigma^{D}
$$

where $c_{1}, c_{2}, \ldots$ are constants and such that $\left\{X^{\mu_{1}}, X^{\mu_{2}}, \ldots, X^{\mu_{n}}\right\}=0$ except for the specific value

$$
L^{2 n}\left\{X^{1}, X^{2}, \ldots, X^{n}\right\}^{2}=\left(c_{1} c_{2} \ldots \ldots c_{n}\right)^{2} L^{2 n}=\left(\frac{d X^{\mu_{1} \mu_{2} \ldots \ldots \mu_{n}}}{d \Sigma}\right)^{2}=|\mathbf{V}|^{2}=\text { constant }
$$

On the other hand, from the equations of motion associated with the free polyparticle action in $C$-space [4], after taking into account that $\left(\frac{d X^{M}}{d \Sigma}\right)^{2}=1$ and that $\Sigma$ is chosen to be dimensionless,

$S=\int d \Sigma=\int d \Sigma \sqrt{\left(\frac{d X^{M}}{d \Sigma}\right)^{2}} \Rightarrow \frac{d^{2} X^{M}}{d \Sigma^{2}}=0, X^{M} \equiv X, X^{\mu}, X^{\mu_{1} \mu_{2}}, \cdots, X^{\mu_{1} \mu_{2} \ldots \mu_{D}}$

one concludes that the components $X^{\mu_{1} \mu_{2} \ldots . . \mu_{n}}(\Sigma)$ grow/decrease linearly with $\Sigma$ so that the $n$-volume velocity components $V^{\mu_{1} \mu_{2} \ldots \mu_{n}}=\frac{d X^{\mu_{1} \mu_{2} \ldots \ldots \mu_{n}}}{d \Sigma}$ are constant. This is indeed consistent with the results found in eq-(20) where the magnitude of the $n$-volume velocity $\mathbf{V}$ is constant. If the $n$-volume velocity components are constant then the magnitude of the $n$-volume velocity is also constant. The converse is not true. A typical example is ordinary circular motion. In [13] it was shown that when the areal velocities are constant the Nambu and Schild string actions lead to equivalent equations of motion. Similar conclusions hold for $p$-brane actions.

Proceeding with eqs- $(19,20)$ one learns, if one equates both sides of eq- $(14 \mathrm{~b})$ and sets $T_{n}=L^{-n}$, that the (dimensionless) polyparticle's proper time in $C$-space obeys

$$
\Sigma=\frac{1}{L^{n}} \int d^{n} \sigma=\frac{\Omega_{n}}{L^{n}}, \quad n=1,2, \ldots, D
$$

and also

$$
|\mathbf{V}| \Sigma=V_{n}=\int d^{n} \sigma \sqrt{\left\{X^{\mu_{1}}, X^{\mu_{2}}, \ldots, X^{\mu_{n}}\right\}^{2}}, \quad n=1,2, \ldots, D
$$

Because the magnitudes of the $n$-volume velocities $|\mathbf{V}|$ are constant, from (22b) one learns that the scale sizes of the evolving world lines, world sheets, world volumes, .... are linearly proportional to the polyparticle's proper time $\Sigma$ in $C$-space. The scale $L$ can be taken to be the Planck length. This is not unlike to cosmological models where the size of the cosmos is taken as a "dilational" clock. From eq-(22a) one may also infer directly that $\Sigma$ only remains invariant under length, area, volume, hyper-volume preserving diffeomorphisms of the $\sigma^{a}$ coordinates. We have chosen a very simple case in eqs-(19). More complicated cases warrant further investigation.

To study further the polyparticle/p-brane correspondence let us begin by writing the wave equation associated with a free polyparticle in $C$-space in natural units $\hbar=c=1$

$$
i \frac{\partial \Psi\left(X^{M}, \Sigma\right)}{\partial \Sigma}=-\frac{\partial^{2} \Psi\left(X^{M}, \Sigma\right)}{\partial X^{M} \partial X_{M}}, \quad X^{M} \equiv X, X^{\mu}, X^{\mu_{1} \mu_{2}}, \cdots, X^{\mu_{1} \mu_{2} \ldots \mu_{D}}
$$


A solution of (23) consistent with the dispersion relations $P_{M} P^{M}=M^{2}$ is

$$
\Psi\left(X^{M}, \Sigma\right)=\operatorname{Exp}\left[i\left(P_{M} X^{M}-M^{2} \Sigma\right)\right]
$$

inserting the solution (24a) into (23) yields $\left(P_{M} P^{M}-M^{2}\right) \Psi=0$, and which in turn, after replacing $P_{M} \rightarrow-i \partial / \partial X^{M}$ leads to the analog of the Klein-Gordon equation in $C$-space for the Clifford-scalar-field $\Phi\left(X^{M}\right)$

$\left(\frac{\partial^{2}}{\partial X^{M} \partial X_{M}}+M^{2}\right) \Phi\left(X^{M}\right)=0$, when $\Psi\left(X^{M}, \Sigma\right)=\Phi\left(X^{M}\right) \operatorname{Exp}\left[-i M^{2} \Sigma\right]$

Choosing an ansatz solution to eq-(23) given by the following "diagonal" sum

$$
\Psi\left(X^{M}, \Sigma\right)=\Psi_{0}(X, \Sigma)+\Psi_{1}\left(X^{\mu}, \Sigma\right)+\Psi_{2}\left(X^{\mu_{1} \mu_{2}}, \Sigma\right)+\ldots+\Psi_{D}\left(X^{\mu_{1} \mu_{2} \ldots \mu_{D}}, \Sigma\right)
$$

and inserting it into eq-(23) leads to the family of decoupled equations

$$
i \frac{\partial \Psi\left(X^{\mu_{1} \mu_{2} \ldots . . \mu_{p+1}}, \Sigma\right)}{\partial \Sigma}=-\frac{\partial^{2} \Psi\left(X^{\mu_{1} \mu_{2} \ldots \mu_{p+1}}, \Sigma\right)}{\partial X^{\mu_{1} \mu_{2} \ldots \mu_{p+1}} \partial X_{\mu_{1} \mu_{2} \ldots \mu_{p+1}}}, \quad p=-1,0,1,2, \cdots, D-1
$$

where $p=-1$ corresponds to the scalar part $X$ of the polyvector $X^{M}$. In the string, $M$-theory literature $p=-1$ corresponds to brane-instantons [1].

We are going to compare the wave equations (26) with the $p$-brane ( $p$-loop) wave functional "Schroedinger-like" equation obtained by [13] and which are based on the Schild $p$-brane actions that are invariant under length, area, volume, hyper-volume preserving diffeomorphisms of the $\sigma^{a}$ coordinates. As a result the corresponding Hamiltonian density is not zero, whereas the Hamiltonian density associated with the fully reparametrization invariant Nambu-Goto $p$-brane action is zero. The $p$-brane ( $p$-loop) wave functional "Schroedinger-like" equation [13] is given by

$$
\begin{gathered}
-\frac{1}{2(p+1) ! m^{p+1}}\left(\oint_{C_{p}} d^{p} \mathbf{s} \sqrt{\left(\mathbf{Y}^{\prime}\right)^{2}}\right)^{-1} \oint_{C_{p}} d^{p} \mathbf{s} \sqrt{\left(\mathbf{Y}^{\prime}\right)^{2}} \frac{\delta^{2} \Psi\left[C_{p} ; V_{p+1}\right]}{\delta Y^{\mu_{0} \mu_{1} \mu_{2} \ldots \mu_{p}}(\mathbf{s}) \delta Y_{\mu_{0} \mu_{1} \mu_{2} \ldots \mu_{p}}(\mathbf{s})}= \\
i \frac{\partial \Psi\left[C_{p} ; V_{p+1}\right]}{\partial V_{p+1}}
\end{gathered}
$$

where $\mathbf{s} \equiv s^{1}, s^{2}, \ldots, s^{p}$ are the intrinsic spatial coordinates of the spatial $p$-loop $C_{p}$ of topology $S^{p}$ (a closed $p$-brane) that is moving in a $D$-dimensional target spacetime background and sweeping a $p+1$-dimensional timelike world hypertube $\Omega_{p+1}$ whose $p+1$ dimensional proper volume is $V_{p+1}$. The proper volume $V_{p+1}$ acts now as a clock/temporal variable and from eq- $(22)$ one can see its relation to the polyparticle's proper time $\Sigma$ in $C$-space.

The $p+1$-vectors $Y^{\mu_{0} \mu_{1} \mu_{2} \ldots \mu_{p}}\left[C_{p}(\mathbf{s})\right]$ are the holographic [13] coordinates associated with the spatial $C_{p}$ loop located at the boundary of the time-like world hypertube after it has swept a $V_{p+1}$ volume. The $C_{p}$-loop encloses a $p+1$-dim region and whose 
projections onto the coordinate planes define the values of the holographic coordinates $Y^{\mu_{0} \mu_{1} \mu_{2} \ldots . \mu_{p}}\left[C_{p}(\mathbf{s})\right]$.

The measure of integration of the $p$-dimensional loop $C_{p}$ of topology $S^{p}$ is given in terms of the square root of

$$
\left(\mathbf{Y}^{\prime}\right)^{2} \equiv\left\{Y^{\mu_{1}}, Y^{\mu_{2}}, \ldots, Y^{\mu_{p}}\right\}^{2}, \quad\left\{Y^{\mu_{1}}, Y^{\mu_{2}}, \ldots, Y^{\mu_{p}}\right\} \equiv \epsilon^{i_{1} i_{2} \ldots . i_{p}} \frac{\partial Y^{\mu_{1}}}{\partial s^{i_{1}}} \frac{\partial Y^{\mu_{2}}}{\partial s^{i_{2}}} \cdots \frac{\partial Y^{\mu_{p}}}{\partial s^{i_{p}}}
$$

where $Y^{\mu}(\mathbf{s})$ are the ordinary coordinates in spacetime of the points of the $p$-loop $C_{p}$.

The terms inside the integrand in the left-hand side of the loop wave equation (27) explicitly depend on the spatial loop coordinates $\mathbf{s}$ at each point of the $p$-loop $C_{p}$, whereas the terms in the right-hand side only depend on the shape of the $p$-loop. For this reason one must integrate the left-hand side along all the points of the $p$-loop. This integration amounts effectively to taking the loop-average of $\frac{\delta^{2} \Psi\left[C_{p} ; V_{p+1}\right]}{\delta Y^{\mu_{0} \mu_{1} \mu_{2} \ldots \mu_{p}}(\mathbf{s}) \delta Y_{\mu_{0} \mu_{1} \mu_{2} \ldots \mu_{p}}(\mathbf{s})}$. In the particular case when the non-zero modes contribution averages to zero, one is solely left with the zero-modes contribution

$$
<\frac{\delta^{2} \Psi\left[C_{p} ; V_{p+1}\right]}{\delta Y^{\mu_{0} \mu_{1} \mu_{2} \ldots \mu_{p}}(\mathbf{s}) \delta Y_{\mu_{0} \mu_{1} \mu_{2} \ldots \mu_{p}}(\mathbf{s})}>_{\text {average }}=\frac{\partial^{2} \Psi\left[Y_{(0)}^{\mu_{0} \mu_{1} \mu_{2} \ldots \mu_{p}} ; V_{p+1}\right]}{\partial Y_{(0)}^{\mu_{0} \mu_{1} \mu_{2} \ldots \mu_{p}} \partial Y_{\mu_{0} \mu_{1} \mu_{2} \ldots \mu_{p} ;(0)}}
$$

such that the wave equation associated with the latter zero-modes denoted by $Y_{(0)}^{\mu_{0} \mu_{1} \mu_{2} \ldots . . \mu_{p}}$ becomes

$$
-\frac{1}{2(p+1) ! m^{p+1}} \frac{\partial^{2} \Psi\left[Y_{(0)}^{\mu_{0} \mu_{1} \mu_{2} \ldots . \mu_{p}} ; V_{p+1}\right]}{\partial Y_{(0)}^{\mu_{0} \mu_{1} \mu_{2} \ldots \mu_{p}} \partial Y_{\mu_{0} \mu_{1} \mu_{2} \ldots \mu_{p} ;(0)}}=i \frac{\partial \Psi\left[Y_{(0)}^{\mu_{0} \mu_{1} \mu_{2} \ldots \mu_{p}} ; V_{p+1}\right]}{\partial V_{p+1}}
$$

and bears now an identical expression (up to numerical factors) to the functional form of eq-(26) obtained from the wave equation of a free polyparticle in $C$-space. This also requires using the explicit correspondence $\Sigma \sim V_{p+1}$ derived in eq- $(22 \mathrm{~b})$, when $n=p+1$, so that $\frac{\partial}{\partial \Sigma} \leftrightarrow \frac{\partial}{\partial V_{p+1}}$ and matching the zero modes coordinates (quenched-like degrees of freedom) $Y_{(0)}^{\mu_{0} \mu_{1} \mu_{2} \cdots \mu_{p}}$ to the Clifford polyvectors $X^{\mu_{1} \mu_{2} \cdots \mu_{p+1}}$.

Instead of recurring to the $p$-brane ( $p$-loop) wave functional "Schroedinger-like" equation [13] the polyparticle/p-brane duality/correspondence at the quantum level should be given in terms of the functional (path) integrals for the partition functions

$$
\int\left[D X^{\mu_{1} \mu_{2} \ldots \ldots \mu_{n}}(\Sigma)\right] e^{i S\left[X^{\mu_{1} \mu_{2} \ldots \ldots \mu_{n}}(\Sigma)\right]} \leftrightarrow \int\left[D X^{\mu}\left(\sigma^{a}\right)\right] e^{i S\left[X^{\mu}\left(\sigma^{a}\right)\right]}, a=1,2,3, \cdots, n=p+1
$$

the crux of exploiting the correspondence (30) is that it may provide another avenue to quantize $p$-branes (a notoriously difficult and unsolved problem) from the more straightforward quantization of the polyparticle in $C$-space. For instance, a preliminary relation between the quantum membrane propagator and Clifford-polyvectors was found in [14].

We conclude by adding some further comments. So far we have only discussed the physics of a free polyparticle and free $p$-branes. It is warranted to introduce interactions. 
The Hamiltonian density for $p$-branes in the presence of an external potential is now given by

$$
\mathcal{H}=\frac{m^{(p+1)}}{2(p+1) !}\left\{X^{\mu_{1}}, X^{\mu_{2}}, \ldots, X^{\mu_{p+1}}\right\}^{2}+V\left(X^{\mu_{1}}, X^{\mu_{2}}, \cdots, X^{\mu_{p+1}}\right)
$$

where $X^{\mu}=X^{\mu}\left(\sigma^{a}\right), a=1,2, \cdots, p+1$. Above, we simply added a potential term to the kinetic terms of the $p$-brane Schild Hamiltonian density.

The polyparticle version of eq-(31) is

$$
\mathcal{H}=\frac{m^{(p+1)}}{2 L^{2(p+1)}(p+1) !}\left(\frac{d X^{\mu_{1} \mu_{2} \cdots \ldots \mu_{p+1}}}{d \Sigma}\right)^{2}+V\left(X^{\mu_{1} \mu_{2} \cdots \mu_{p+1}}\right)
$$

One may choose for potential (density) $V$ the polyparticle analog of the relativistic point-particle harmonic oscillator and whose wave-functions in both configuration and Bargmann-Fock like space were found using group-theoretical methods by [15]. These wave-functions are provided by generalized Hermite polynomials. Similarly, one may explore the polyparticle generalization in $C$-space of the Born-Dirac oscillator [16] which is now characterized by an equation of the form

$$
\left(i \gamma^{M} \frac{\partial}{\partial X^{M}}+i \lambda \gamma^{M} X_{M}-\mathcal{M}\right) \Psi\left(X^{M}, \Sigma\right)=i \frac{\partial \Psi}{\partial \Sigma}, X^{M} \equiv X, X^{\mu}, X^{\mu_{1} \mu_{2}}, \cdots, X^{\mu_{1} \mu_{2} \ldots \mu_{D}}
$$

and exhibiting an $X^{M} \leftrightarrow P^{M}$ Born's reciprocity symmetry. As usual, powers of a suitable length (inverse mass) must be inserted in (33) to match physical units. The quantization of the Born-Dirac polyparticle oscillator in $C$-spaces (construction of the explicit quantum states, spectrum, ....) may facilitate the quantization program of $p$-branes living in ordinary spacetimes, and experiencing an external interaction, via the $p$-brane/polyparticle duality/correspondence proposed in this work. The energy-angular momentum spectrum of the Born-Dirac point-particle oscillator behaves as $J \sim E^{2}[16]$ which resembles the Regge behavior of the string.

Another Schroedinger wave functional equations worth mentioning are those based on the De Donder-Weyl quantization approach to gravity [17] where a parameter of inverse spatial volume dimensions, Clifford-valued wave functions and Clifford-Dirac operators are essential. To finalize, and related to the quantization approach of $p$-branes via the quantum polyparticle, it is worth mentioning that the $n$-ary commutators of the quantum operators $\hat{X}^{\mu}$ can be expressed in terms of Clifford polyvector-valued coordinate operators as follows

$$
\left[\hat{X}^{\mu_{1}}, \hat{X}^{\mu_{2}}\right] \sim \hat{X}^{\mu_{1} \mu_{2}},\left[\hat{X}^{\mu_{1}}, \hat{X}^{\mu_{2}}, \hat{X}^{\mu_{3}}\right] \sim \hat{X}^{\mu_{1} \mu_{2} \mu_{3}}, \ldots,\left[\hat{X}^{\mu_{1}}, \hat{X}^{\mu_{2}}, \cdots, \hat{X}^{\mu_{p+1}}\right] \sim \hat{X}^{\mu_{1} \mu_{2} \cdots \mu_{p+1}}
$$

when the coordinate algebra is isomorphic to the Clifford algebra [18]. The essence of (34) is the compositeness nature of the polyvector-valued coordinate operators in terms of the ordinary $p$-brane coordinate operators.

\section{Acknowledgments}


We are indebted to M. Bowers for assistance and to Igor Kanatchikov for correspondence.

\section{References}

[1] R. Blumenhagen, D. Lust and S. Theisen, Basic Concepts of String Theory (Springer 2013).

P. West, Introduction to Strings and Branes (Cambridge University Press 2012).

K. Becker, M. Becker and J. Schwartz, String-Theory and M-theory: A Modern Introduction (Cambridge University Press 2007).

[2] O. Hohm and H. Samtleben, "Exceptional Field Theory III" Phys. Rev. D 90, 066002 (2014) [arXiv : 1406.3348].

[3] G. Papadopoulos, "C-spaces, generalized geometry and double field theory" arXiv : 1412.1146

[4] C. Castro and M. Pavsic, "The Extended Relativity Theory in Clifford-spaces", Progress in Physics, vol. 1 (2005) 31. Phys. Letts B 559 (2003) 74. Int. J. Theor. Phys 42 (2003) 1693.

[5] C. Castro, "The Extended Relativity Theory in Clifford Phase Spaces and Modifications of Gravity at the Planck/Hubble scales" , Advances in Applied Clifford Algebras 24 (2014) 29.

[6] D. Hestenes, Spacetime Algebra (Gordon and Breach, New York, 1996).

D. Hestenes and G. Sobcyk, Clifford Algebra to Geometric Calculus (D. Reidel Publishing Company, Dordrecht, 1984).

[7] M. Pavsic, Found. of Phys. 33 (2003) 1277.

M. Pavsic, "The Landscape of Theoretical Physics : A Global View, from point particles to the brane world and beyond, in search of a Unifying Principle'", (Fundamental Theories of Physics, vol. 19, Kluwer Academic Publishers, Dordrecht, Boston, London, 2001).

[8] M. Pavsic, J. Phys. A41 :332001, (2008).

[9] C. Castro, "Extended Lorentz Transformations in Clifford Space Relativity Theory", to appear in Advances in Applied Clifford Algebras.

[10] C. Castro, "Novel Physical Consequences of the Extended Relativity in Clifford Spaces", to appear in Advances in Applied Clifford Algebras.

[11] A. Cayley, Cambridge Math. J. 4 (1845) 193. 
[12] I. Gelfand, M. Kapranov and A. Zelevinsky, Discriminants, Resultants and Determinants (Birkhauser 1994).

[13] S. Ansoldi, "Boundary versus Bulk Dynamics of Extended Objects and the Fractal Structure of Quantum Spacetime" arXiv: hep-th/0403066 (Ph.D Thesis, University of Trieste, Italy 2004).

S. Ansoldi, A. Aurilia and E. Spallucci, Class. Quan. Grav 19 (2002) 3207.

S. Ansoldi, C. Castro and E. Spallucci, Class. Quan. Grav 18 (1999) 1833.

[14] S. Ansoldi, A. Aurilia, C. Castro and E. Spallucci, Phys. Rev. D 64 (2001) 0260003.

[15] V. Aldaya, J. Bisquert, J. Guerrero and J. Navarro-Salas, Reports on Mathematical Physics 37, no. 3 (1996) 387.

V. Aldaya, J . Bisquert and J. Navarro-Salas, Phys. Letts A 156 (1991) 381.

[16] M. Moshinsky and A. Szczepaniak, J. Phys A : Math. Gen. 22 (1989) L817.

O. de Lange, J. Phys A : Math. Gen. 24 (1991) 667.

[17] I.V. Kanatchikov, "On the precanonical structure of the Schroedinger wave functional" arXiv : 1312.4518.

I.V. Kanatchikov. "De Donder-Weyl Hamiltonian formulation and precanonical quantization of vielbein gravity" J. Phys.: Conf. Ser. 442012041 (2013), arXiv : 1302.2610 .

I.V. Kanatchikov, "On precanonical quantization of gravity" arXiv : 1407.3101.

[18] C. Castro, "On N-ary Algebras, Branes and Polyvector-valued Gauge Theories in Noncommutative Clifford Spaces"; J. Phys A : Math. Theor. 43 (2010) 365201. 